20. Kane MT (1992) An argument-based approach to validity. Psychol Bull 112:527

21. Kazdin AE (1977) Artifact, bias, and complexity of assessment: The ABCs of reliability. J Appl Behav Anal 10:141

22. Keefe FJ, Block AR (1982) Development of an observation method for assessing pain behavior in chronic low back pain patients. Behav Ther 13:363

23. Keijsers JF, Steenbakkers MW, Gerards FM, Meertens RM (1990) The efficacy of the back school: An analysis of the literature. Arthritis Care Res 3:210

24. Keijsers JF, Steenbakkers MW, Meertens RM, Bouter LM, Kok G (1990) The efficacy of the back school: A randomized trial. Arthritis Care Res 3:204

25. Kröner-Herwig B (1990) Chronischer Schmerz - Eine Gegenstandsbestimmung. In: Basler HD, Franz C, Kröner-Herwig B, Rehfisch HP, Seemann H (Hrsg) Psychologische Schmerztherapie. Springer, Berlin Heidelberg New York, S 1

26. Lankhorst GJ, Van de Stadt RJ, Van der Korst JK (1985) The natural history of idiopathic low back pain. Scand J Rehabil Med 17:1

27. Lienert GA (1967) Testaufbau und Testanalyse, 2. Aufl. Beltz, Weinheim

28. Mattmiller AW (1980) The California Back School. Physiotherapy $66: 118$

29. Mitchell SK (1979) Interobserver agreement, reliability, and generalizability of data collected in observational studies. Psychol Bull 86:376
30. Nentwig CG, Czolbe AB (1990) Methoden and Ergebnisse der Evaluation. In: Nentwig CG, Krämer J, Ullrich CH (Hrsg) Die Rückenschule. Enke, Stuttgart, S 97

31. Nentwig CG, Ullrich CH (1990) Wirksamkeit eines Verhaltenstrainings für Wirbelsäulenpatienten: Eine prospektive kontrollierte Studie. In: Nentwig CG, Krämer J, Ullrich CH (Hrsg) Die Rückenschule. Enke, Stuttgart, S 103

32. Seemann H, Zimmermann M (1990) Kybernetische Schmerzkonzepte - Eine Standortbestimmung. In: Basler HD, Franz C, Kröner-Herwig B, Rehfisch HP, Seemann H (Hrsg) Psychologische Schmerztherapie. Springer, Berlin Heidelberg New York, S 17

33. Suen HK (1988) Agreement, reliability, accuracy, and validity: Toward a clarification. Behav Assess 10:343

34. Suen HK, Ary D (1989) Analyzing quantitative behavioral observation data. Erlbaum, Hillsdale, NJ

35. Taplin PS, Reid JB (1973) Effects of instrumental set and experimenter influence on observer reliability. Child Dev 44:547

Prof. Dr. H.-D. Basler

Institut für Medizinische Psychologie

Fachbereich Humanmedizin

Philipps-Universität

Bunsenstraße 3

W-3550 Marburg

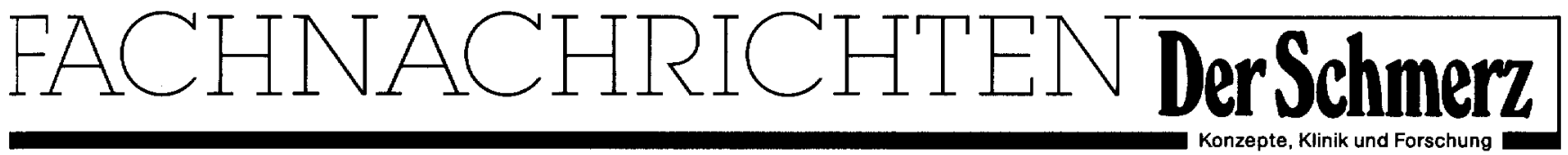

\section{Mitgliedschaft in der International Society for the Study of Pain (IASP)}

Nicht alle Mitglieder der Deutschen Gesellschaft zum Studium des Schmerzes wissen, daß unsere Gesellschaft ursprünglich als „Chapter" der International Association for the Study of Pain (IASP) entstanden ist. Man kann Mitglied unserer Schmerzgesellschaft sein, ohne der IASP anzugehören.

Welchen Sinn hat eine Zugehörigkeit zur IASP? Ein eigener Mitgliedsbeitrag ist zu entrichten, der jährlich $\$ 80$ oder mehr beträgt, je nach Einkommen (Selbsteinschätzung). Für diesen Beitrag erhält jedes IASP Mitglied ein Abonnement der Zeitschrift „Pain“. Außerdem veranstaltet die IASP alle 3 Jahre einen wissenschaft- lichen Weltkongreß. Der vorletzte fand 1987 in Hamburg statt, der nächste im August 1993 in Paris.

Wer sollte Mitglieder der IASP werden? Ich kann mir vorstellen, daß manche Schmerztherapeuten, die ausschließlich an der praktisch-klinischen Entwicklung des Faches interessiert sind, mit dem Fortbildungsangebot und der Interessenvertretung durch die ,Deutsche Gesellschaft zum Studium des Schmerzes" voll befriedigt sind. Wer allerdings an der wissenschaftlichen Diskussion in der Schmerzforschung teilhaben will, für den ist die IASP ein unverzichtbares Forum. Einen aktiven Beitrag bei den Weltkongressen dürfen nur
Mitglieder einbringen, oder Wissenschaftler, die von einem Mitglied gesponsort werden.

Ich möchte daher nochmals - wie schon bei unserem schönen Jahreskongre $\beta$ in Berlin - bei den wissenschaftlich interessierten Mitgliedern der „Deutschen Gesellschaft zum Studium des Schmerzes" für einen Beitritt zur IASP werben. Anträge können Sie beim Sekretariat unserer Gesellschaft, bei Prof. Zimmermann oder bei mir anfordern. Prof. Zimmermann und ich sind auch gerne bereit, die für Neumitglieder erforderlichen Bürgschaftsunterschriften $\mathrm{zu}$ leisten.

Prof. Dr. H.O. Handwerker Institut für

Physiologie \& Biokybernetik Universitätsstraße 17

W-8520 Erlangen 